\title{
Comunicação alternativa e ampliada para educandos com autismo: CONSIDERAÇÕES METODOLÓGICAS
}

A UGMENTATIVE AND ALTERNATIVE COMMUNICATION FOR INDIVIDUALS WITH AUTISM: METHODOLOGICAL CONSIDERATIONS

Débora Regina de Paula NUNES ${ }^{1}$ Francisco de Paula NUNES SOBRINHO²

\begin{abstract}
RESUM O: foram analisadas as características metodológicas de 56 artigos científicos focados no tema Comunicação Alternativa e Ampliada (CAA), para educandos com espectro do autismo. Esses artigos foram produzidos no período 1980-2007; 18 versaram sobre o uso de sistemas manuais e língua de sinais; 26 empregaram sistemas pictográficos de comunicação; 9 utilizaram sistemas assistidos com acionadores de voz e 3 utilizaram sistemas híbridos, contendo mais de uma modalidade de CAA. Com base nos resultados desta análise preliminar, a proposta foi direcionada para a identificação das melhores práticas adotadas pelos programas de intervenção para esta população. Os resultados da análise das características metodológicas dos artigos revisados sustenta a predominância de delineamentos experimentais e quase-experimentais do tipo intrassujeitos, o que corrobora a escassez de pesquisas de grupo em população de autistas. Os estudos envolveram, predominantemente, número reduzido de participantes, selecionados deforma incidental. Metade das pesquisas revisadas descreve, claramente, medidas de generalização de comportamentos, para ambientes naturais ou sustentabilidade de respostas após a intervenção. Foram escassos os estudos avaliativos do grau de satisfação dos participantes no que se refere aos efeitos produzidos pela intervenção. N esta revisão foram identificadas as melhores práticas no atendimento da população autista, tendo como fundamento a validade interna, a validade externa e a validade social das intervenções. Indicações de diretrizes válidas e confiáveis foram apontadas no sentido de favorecerem o desenvolvimento de projetos científicos sobre o tema.
\end{abstract}

PA LA V RAS-CH AVE: educação especial; autismo; comunicação al ternativa eampliada; metodologia científica; revisão de literatura.

A BSTRACT: The methodological characteristics of fifty-six studies published between 1980 and 2007 involving the use of augmentative and alternative communication (AAC) by individuals with autism spectrum disorder (ASD) were analyzed. Eighteen investigations implemented sign language or manual signs, twenty-six used picture-based systems, nine employed voice output devices and three worked with hybrid systems, combining two or moreAAC modalities. This review focused on identifying the best practices implemented by intervention programs for individuals with ASD, considering measures of internal, external and social validity. A predominance of experimental and quasi-experimental single subject research designs were identified, whereas a limited number of studies employed group designs. Most worked with a reduced number of participants, which were mainly selected in incidental manners. Half of the investigations clearly revealed generalization measures to other behaviors and environments, as well as the sustainability of the results post intervention. A

${ }^{1}$ Ph.D. em Educação Especial pela Florida StateUniversity, Professora A djunta do Departamento de Educação/ Programa de Pós-graduação em Educação da Universidade Federal do Rio Grande do Norte deboranunes@ufrnet.br

2 Ph.D. em Educação Especial pela Vanderbilt University, Professor Titular do Departamento de Estudos da Educação Inclusiva e Continuada/ Programa de Pós-graduação em Políticas Públicas e Formação Humana da Universidade do Estado do Rio de Janeiro - fnunessobrinho@yahoo.com.br 

for developing intervention studies with reliable results was further discussed.

KEYWORD S: Autism; Augmentative and Alternative Communication; Research Methodology; Literature Review.

\section{NTRODUÇÃo}

O objetivo do presenteartigo éanalisar as características metodológicas do acervo constituído de 56 trabalhos científicos focados na Comunicação Alternativa e Ampliada (CAA), para educandos com autismo, que foram produzidos no período $1980-2007^{3}$. Com base nos resultados desta análise preliminar, a proposta foi direcionada para a identificação das melhores práticas adotadas pelos programas de intervenção para esta população. No dizer de Spaulding (2009) as melhores práticas, em Educação Especial, se constituem em modalidades de intervenções mais eficazes e eficientes e que asseguram um desempenho ótimo do aluno. Como complemento do presente artigo, são traçadas diretrizes que favoreçam o desenvolvimento de projetos de pesquisas cientificamente válidos sobre o assunto.

Inicialmente, vale assinalar que Comunicação Alternativa e Ampliada (CAA) está sendo conceituada neste artigo como uma ampla variedade de métodos e técnicas que complementam ou substituem a linguagem oral comprometida ou ausente. Em uma avaliação de programas de intervenção direcionados para indivíduos com autismo, o uso da CAA é categorizado como prática promissora (SIMPSON , 2005). Ou seja, os resultados das pesquisas conduzidas sob esteenfoque evidenciam a eficácia da CAA para esta população, embora sejam necessários estudos que evidenciem maior rigor metodológico, a fim de que a CAA seja conceituada como prática cientificamente válida4 (SIMPSON, 2005).

Os resultados de uma pesquisa podem contribuir, significativamente, para a produção, discussão e dissemi nação do conhecimento científico desde que sejam experimentalmente válidos. Embora a validade experimental que se busca em termos de validade interna e validade externa seja um ideal pretendido pelo pesquisador, équase improvável que isso ocorra. A validade interna émuito difícil de ser obtida em ambientes externos ao laboratório de experimentos comportamentais nos quais se observa outras variáveis estranhas que se tenta controlar (BEST; KAHN, 2006). Para estes autores, quando os controles

\footnotetext{
${ }^{3}$ Os dados apresentados no presente artigo são derivados do estudo: NUNES, D. AAC Interventions for autism: A research summary. International Journal of Special Education. v.23, p.17 - 26, 2008.

${ }^{4}$ Práticas cientificamenteváli idas são definidas como métodos etécnicas quetenham satisfeito padrões científicos rigorosos determinados por múltiplos revisores e que, quando replicados de forma consistente, válida e fidedigna, produzido resultados positivos.
} 
experimentais são muito rígidos, no sentido de se alcançar a validade interna, mais artificial e menos realística é a situação que pode prevalecer, reduzindo assim a validade externa ou possi bilidade degeneralização do experimento. N estecontexto, é instituída a concepção de demanda de produção de pesquisas que garantam adequados níveis de val idade interna, validade externa evalidade social ( $N$ ational Research Council, NRC, 2001).

Para que um experimento seja considerado internamente válido é preciso queapresente resultados com pouca ambiguidade ou nenhuma chance de dupla interpretação (NUNES SOBRINHO; NAUJORKS, 2001). De acordo com o NRC (2001), os estudos que evidenciam maior validade interna são os que comparam os efeitos de diferentes programas de intervenção ou que confrontam os resultados do tratamento com procedimentos placebo. Em seguida são consideradas as investigações que fazem uso dos delineamentos de pesquisa experimentais intrassujeitos, também denominadas experimentos de caso-único (KEN NEDY, 2005). Trata-se de modal idades de arranjos especiais de pesquisa que permitem demonstrar os efeitos de uma série de mani pulações experimentais em um único indivíduo. Neste tipo de delineamento de pesquisa os aspectos individuais são valorizados em detrimento da comparação dos níveis de desempenho entre indivíduos pertencentes a um mesmo grupo investigado (NUNESSOBRINHO; NAUJORKS, 2001).

Dentre os del ineamentos de pesquisa experimentais intrassujeitos que permitem mai or controle da variável independente destacam-se os delineamentos do tipolinha de base múltipla , os do tipo reversivo (A-B-A-B) edo tipo alternado ( $A$ B-C) (NRC, 2001). No primeiro caso a variável independente (programa de treinamento) éintroduzida em ocasiões diferentes para os diversos participantes/ contextos com o objetivo de modificar seus respectivos comportamentos (variável dependente) para demonstrar deforma mais clara seus efeitos (NUNES et al., 1998). No segundo caso, a intervenção é aplicada de modo sequencial e retirada para testagem dos efeitos do tratamento sobre o comportamento-alvo (NUNES SOBRINHO; NAUJORKS, 2001). No delineamento de pesquisa intrassujeitos do tipo alternado, os efeitos de duas ou mais condições de tratamento (B e C) sobre um único comportamento são examinadas (KEN NEDY, 2005).

De acordo com o NRC (2001) os delineamentos de pesquisa de grupo constituídos de pré e de pós-teste com aval iações independentes são igualmente robustos, permitindo inferir com maior segurança, que a intervenção (variável independente) foi a única responsável pelas mudanças ocorridas nas respostas produzidas (variável dependente). Em terceiro lugar, o NRC (2001) cita os estudos quase-experimentais que comportam, por exemplo, delineamentos do tipo A-B. No caso, a variável dependente é mensurada repetidamente sob controle da linha de base (A) e da intervenção (B) (NUNES SOBRINHO; NAUJORKS, 2001). N este tipo de delineamento de pesquisa o controle experimental é inferior aos delineamentos do tipo linha de base múltipla ou do tipo reversivo (KEN NEDY, 2005). Por fim, em uma categoria denominada "outros", estariam agrupados os 
estudos descritivos que não objetivam o controle experimental, mas a descrição aprofundada e exaustiva de um ou poucos objetos (GIL, 2007).

O conceito deval idade externa refere-se ao fato de que as relações entre variáveis (relações de causa efeito) podem ser generalizadas para outros ambientes ou locais, para outras variáveis tratamento, para outras medidas de variáveis e para outras populações ou participantes da pesquisa. Quando se trata de validade externa, pel o menos dois aspectos devem ser considerados. O primeiro diz respeito à eficácia da intervenção ou tratamento. Na medida em que uma intervenção específica produz efeito no comportamento dos participantes, será que produzirá também o mesmo efeito com outros participantes, em outros ambientes, quando conduzida por outro pesquisador equando ocorrerem modificações discretas nos procedimentos originais da pesquisa?

\begin{abstract}
Há validade externa quando a pesquisa de avaliação demonstra algo que é verdadeiro para além dos estreitos limites do seu estudo. Seos resultados forem verdadeiros não apenas para o momento, lugar e pessoas de um certo estudo, mas também o forem para outros momentos lugares e pessoas, o estudo possuirá validade externa. Esta pode ser obtida pela replicação da pesquisa, testando-se a coerência entre os achados do estudo e os resultados de outras investigações semelhantes (MORAIS, 2007, p.72).
\end{abstract}

De acordo com o NRC (2001), grupos randomicamente selecionados com tamanhos amostrais adequados propiciam os melhores níveis de validade externa. A pesar de a randomização favorecer o controle de variáveis estranhas (COZBY, 2003) é importante ressaltar que, em muitos casos, a seleção estocástica deamostras envolvendo participantes com autismo é infactível (NRC, 2001). Este fato se justifica pela diversidade de características dos indivíduos diagnosticados com a síndrome, a heterogeneidade dos programas de intervenção, ea necessidade dese ajustar o del ineamento de pesquisa às questões propostas pelo estudo (NRC, 2001; SIMPSON , 2005).

Assim, o NRC (2001) assume que os grupos não randomizados, mas com amostras que evidenciam claros critérios de inclusão/ exclusão podem propiciar bons níveis de validade externa. N esta categoria estão também presentes os delineamentos de pesquisa do tipo sujeito-único que tenham sido replicados por, ao menos, três vezes consecutivas. Por fimencontram-se pesquisas envolvendo três ou mais participantes incidentalmente selecionados em delineamentos experimentais e quase-experimentais intrassujeitos (NRC, 2001).

A validade social implica na generalização de resultados deambientes artificiais para ambientes naturais (NRC, 2001), na sustentabilidade dos efeitos de uma intervenção, como também no grau de significância social das mudanças produzidas na perspectiva do consumidor - ou seja, os indivíduos afetados pelos resultados da pesquisa - (KEN N EDY, 2005). O que se pretende é verificar o grau de funcionalidade dos resultados da investigação científica quando aplicados no 
contexto social. Inicialmente proposto por (WOLF, 1978), o conceito de validade social emerge por conta das necessidades práticas dos anal istas de comportamento em tomar conhecimento das implicações e da relevância dos resultados das suas próprias pesquisas para os membros de uma sociedade. N este contexto, sustentabilidade ou manutenção de respostas é compreendida como o prosseguimento na emissão de um comportamento ou habilidade desenvolvida durante um tratamento, após a finalização de um programa de intervenção (NRC, 2001; KEN NEDY, 2005).

Ao referir-se à natureza aplicada da pesquisa educacional, Kennedy (2005) sustenta a necessidade dese avaliar também o grau de satisfação das pessoas - os consumidores da pesquisa - com relação aos efeitos produzidos em uma ampla variedade de segmentos da sociedade. Esta atividade analítica adicional atribuída ao pesquisador implica na coleta de informaç̃es junto a esses consumidores ou pessoas afetadas pel os resultados da investigação. À guisa deexemplo, (KENNEDY, 2005) relata uma pesquisa cujo objetivo original écompreender/ verificar o impacto dos resultados da intervenção em uma sala deaula. No caso, adicionalmente, devem ser formuladas perguntas do tipo 0 professor considerou a intervenção fácil ou difícil de ser implementada? Como reagiram os al unos frente ao novo procedimento? 0 professor continuou se utilizando dos procedimentos de intervenção, mesmo depois de concluída a pesquisa?

\section{Desenvolvimento}

A seguir serão descritos aspectos e características metodológicas contidas em 56 estudos produzidos no período 1980-2007 e que registram a utilização da CAA em populações com autismo, conformeresumo apresentado no Quadro 1, considerando-seas modalidades desistemas CAA empregados, os tipos de delineamentos de pesquisa, o número de participantes envolvidos, variáveis independentes evariáveis dependentes, medidas degeneralização, manutenção e de val idade social, conforme abaixo. 


\begin{tabular}{|c|c|}
\hline Autores & $\begin{array}{l}\text { Tipo de CAA, delineamento, número de participantes, variáveis, generalização/ manutenção } \\
\text { de respostas; medidas de satisfação }\end{array}$ \\
\hline arrera e Sulzer- & $\begin{array}{l}\text { SL; Experimental alternado, } 1 \text { participante, comparar efeitos da comunicação total e língua de } \\
\text { sinais na linguagem expressiva usando procedimentos da análise experimental do comportamento } \\
\text { (AEC); GM - III; medida de satisfação não fornecida ou não aplicável }\end{array}$ \\
\hline $\begin{array}{l}\text { Barrera } \\
1980\end{array}$ & $\begin{array}{l}\text { SL; Experimental alternado, } 3 \text { participantes, comparar efeitos da comunicação total e língua de } \\
\text { sinais na linguagem expressiva usando procedimentos AEC; GM - II; medida de satisfação não } \\
\text { fornecida ou não aplicável sequencial }\end{array}$ \\
\hline $\begin{array}{l}\text { Bartman e } \\
\text { Freeman, 2003 }\end{array}$ & $\begin{array}{l}\text { SL; Quase-experimental A-B, } 1 \text { participante, aprendizagem de gestos; GM - III; medida de } \\
\text { satisfação não fornecida ou não aplicável }\end{array}$ \\
\hline $\begin{array}{l}\text { Bernard -Opiz et } \\
\text { al., } 1999\end{array}$ & $\begin{array}{l}\text { VOCA; Grupo comparativo, } 10 \text { participantes, avaliar efeitos de um CAI (computer assisted } \\
\text { instruction) e instrução verbal na frequência de imitação vocal; GM - III; medida de satisfação } \\
\text { fornecida }\end{array}$ \\
\hline $\begin{array}{l}\text { Bondy e Frost, } \\
1994\end{array}$ & $\begin{array}{l}\text { SP; Estudo de caso descritivo, } 1 \text { participante, avaliar efeitos do Picture Exchange Communication } \\
\text { System (PECS) na linguagem expressiva;GM - III; medida de satisfação não fornecida ou não } \\
\text { aplicável }\end{array}$ \\
\hline $\begin{array}{l}\text { Bosseler } \\
\text { Massaro, } 2003\end{array}$ & $\begin{array}{l}\text { Pré e pós teste, } 8 \text { participantes, avaliar efeitos de um software na aquisição de vocabulário; } \\
\text { medida de satisfação não fornecida ou não aplicável }\end{array}$ \\
\hline $\begin{array}{l}\text { Bryan e Gast, } \\
2000\end{array}$ & $\begin{array}{l}\text { de sistema pictográfico na linguagem } \\
\text { fornecida }\end{array}$ \\
\hline Buffington et al., & $\begin{array}{l}\text { ntos da análise experimental do } \\
\text { GM - I; medida de satisfação }\end{array}$ \\
\hline Cafiero, 2001 & $\begin{array}{l}\text { ALS) na } \\
\text { o aplicável }\end{array}$ \\
\hline $\begin{array}{l}\text { Carr e Dores, } \\
1981\end{array}$ & $\begin{array}{l}\text { SL; Linha de ba } \\
\text { total na linguage }\end{array}$ \\
\hline $\begin{array}{l}\text { Carr } \\
\text { Kologinsky, } 1983\end{array}$ & $\begin{array}{l}\text { a AEC na } \\
\text { aplicável }\end{array}$ \\
\hline Carr et al., 1987 & $\begin{array}{l}\text { múltipla, } 4 \text { participantes, avaliação de procedimentos da AEC na linguagem } \\
\text { inais); GM - II; medida de satisfação não fornecida ou não aplicável }\end{array}$ \\
\hline $\begin{array}{l}\text { Carr, Pridal } \\
\text { Dores, } 1984\end{array}$ & $\begin{array}{l}\text {; Grupos comparativos, } 10 \text { participantes, comparar alunos com boa habilidade de imitação } \\
\text { rbal e pouca habilidade de imitação usando língua de sinais e fala; GM - III; medida de } \\
\text { isfação não fornecida ou não aplicável }\end{array}$ \\
\hline $\begin{array}{l}\text { Charlop-Christy } \\
\text { et al., 2002 } \\
\text { Legenda: Sistemas } \\
\text { de voz (VOCA); S } \\
\text { em pelo menos um } \\
\text { Não avaliado ou ou }\end{array}$ & $\begin{array}{l}\text { lagem expressiva e } \\
\text { tidos com acionadores } \\
\text { tervenção observados }\end{array}$ \\
\hline $\begin{array}{l}\text { Cummings e } \\
\text { Williams, } 2000\end{array}$ & $\begin{array}{l}\text { SP; Experimental intrassujeito A-B-C, } 5 \text { participantes, avaliar efeitos do PECS, imitação vocal e } \\
\text { AEC na linguagem expressiva; GM - III; medida de satisfação não fornecida ou não aplicável }\end{array}$ \\
\hline Dyches, 1998 & $\begin{array}{l}\text { Experimental reversivo } \mathrm{ABAB}, 2 \text { participantes, avaliar efeitos de um sistema com } \\
\text { r de voz na linguagem expressiva; GM - III; medida de satisfação não fornecida ou não }\end{array}$ \\
\hline $\begin{array}{l}\text { Ferrarese } \\
\text { Norton, } 1982\end{array}$ & $\begin{array}{l}\text { SL; Estudo comparativo, } 1 \text { participante, comparar articulação da fala usando língua de sinais e sem } \\
\text { usar língua de sinais; GM - III; medida de satisfação não fornecida ou não aplicável }\end{array}$ \\
\hline Frea et al., 2001 & iva e \\
\hline $\begin{array}{l}\text { Ganz, Simpson } \\
2004\end{array}$ & $\begin{array}{l}\text {, avaliar efeitos do PECS na linguagem expressiva; } \\
\text { ou não aplicável }\end{array}$ \\
\hline Ganz et al, 2007 & $\begin{array}{l}\text { SP; Linha de base múltipla, } 3 \text { participantes, } 1 \text { participante, avaliar efeitos do PECS na linguagem } \\
\text { expressiya; GM - III; medida de satişação não fornecida ou não aplicável }\end{array}$ \\
\hline $\begin{array}{l}\text { Hamilton e SnelI, } \\
1993\end{array}$ & $\begin{array}{l}\text {; Linha de base múltipla, } 1 \text { participante, avaliar efeitos de procedimentos naturalísticos de ensino } \\
\text { ivro de comunicação na linguagem expressiva; GM - II; medida de satisfação fornecida }\end{array}$ \\
\hline Keen et al., 2001 & \\
\hline Kouri, 1988 & $\begin{array}{l}\text {; Experimental A-B-A, } 1 \text { participante, avaliar efeitos de procedimentos naturalísticos de ensino e } \\
\text { nunicação total na linguagem expressiva; GM - III; medida de satisfação não fornecida ou não } \\
\text { icável }\end{array}$ \\
\hline
\end{tabular}




\begin{tabular}{|c|c|}
\hline Autores & $\begin{array}{l}\text { Tipo de CAA, delineamento, número de participantes, variáveis, generalização/ manutenção } \\
\text { de respostas; medidas de satisfação }\end{array}$ \\
\hline Kozleski, 1991 & $\begin{array}{l}\text { SH; Linha de base múltipla, } 4 \text { participantes, avaliar efeitos reforçamento contingente na linguagem } \\
\text { expressiva; GM - III; medida de satisfação não fornecida ou não aplicável }\end{array}$ \\
\hline $\begin{array}{l}\text { Kravits et al., } \\
2002\end{array}$ & $\begin{array}{l}\text { SP; Linha de base múltipla, } 1 \text { participante, avaliar efeitos do PECS + treino de habilidades sociais } \\
\text { na linguagem expressiva; GM - II; medida de satisfação não fornecida ou não aplicável }\end{array}$ \\
\hline Layton, 1988 & $\begin{array}{l}\text { SL; Grupos comparativos, } 60 \text { participantes, comparar efeitos de língua de sinais, comunicação total } \\
\text { e fala na linguagem expressiva e receptiva; GM - II; medida de satisfação não fornecida ou não } \\
\text { aplicável }\end{array}$ \\
\hline Light et al., 1998 & $\begin{array}{l}\text { SH; Estudo de caso descritivo, } 1 \text { participante, analise do uso de sistema pictográfico, sistema com } \\
\text { acionador de voz e gestos; GM - II; medida de satisfação fornecida }\end{array}$ \\
\hline $\begin{array}{l}\text { MacDuff et al., } \\
1993\end{array}$ & $\begin{array}{l}\text { SP; Linha de base múltipla, } 4 \text { participantes, avaliar efeitos de calendário visual + modelo com dicas } \\
\text { na linguagem receptiva e comportamento; GM - I; medida de satisfação não fornecida ou não } \\
\text { aplicável }\end{array}$ \\
\hline $\begin{array}{l}\text { Magiati e } \\
\text { Howlin, } 2003\end{array}$ & $\begin{array}{l}\text { SP; Pré e pós teste, } 34 \text { participantes, programa de treinamento de professores usando PECS, efeitos } \\
\text { do programa no desenvolvimento da linguagem expressiva de alunos com autismo } \\
\text { GM - III; medida de satisfação fornecida }\end{array}$ \\
\hline $\begin{array}{l}\text { Marckel, et al, } \\
2006\end{array}$ & $\begin{array}{l}\text { SP; Linha de base múltipla, } 2 \text { participantes, sistema pictográfico na linguagem expressiva; } \\
\text { GM - II; medida de satisfação não fornecida ou não aplicável }\end{array}$ \\
\hline $\begin{array}{l}\text { Nunes and } \\
\text { Hanline, } 2007\end{array}$ & $\begin{array}{l}\text { SP; Linha de base múltipla, } 1 \text { participante, avaliar efeitos do ensino naturalístico + sistema } \\
\text { pictográfico na linguagem expressiva; GM - II; medida de satisfação não fornecida ou não } \\
\text { aplicável }\end{array}$ \\
\hline $\begin{array}{l}\text { O'Neill e } \\
\text { Sweetland- } \\
\text { Baker, 2001 }\end{array}$ & $\begin{array}{l}\text { SP; Experimental alternado, } 2 \text { participantes, avaliar efeitos do Functional Communication Training } \\
\text { na linguagem expressiva e comportamentos disruptivos; GM - I; medida de satisfação não } \\
\text { fornecida ou não aplicável }\end{array}$ \\
\hline $\begin{array}{ll}\text { Parsons e La } \\
\text { Sorte, } 1993\end{array}$ & $\begin{array}{l}\text { VOCA; Experimental alternado, } 6 \text { participantes, avaliar efeitos de um sistema com acionador de } \\
\text { voz na linguagem expressiva; GM - III; medida de satisfação não fornecida ou não aplicável }\end{array}$ \\
\hline \multicolumn{2}{|c|}{$\begin{array}{l}\text { Legenda: Sistemas manuais e/ou língua de sinais (SL); Sistemas pictográficos (SP); sistemas assistidos com acionadores } \\
\text { de voz (VOCA); Sistemas híbridos (SH). Generalização; manutenção de respostas: Efeitos da intervenção observados } \\
\text { em pelo menos um ambiente natural (GM-I.); Generalização para outro ambiente, comportamento ou pessoa; GM-II. } \\
\text { Não avaliado ou outro (GM- III); }\end{array}$} \\
\hline $\begin{array}{l}\text { Peterson et al., } \\
1995\end{array}$ & $\begin{array}{l}\text { SP; Experimental reversivo A-AB-B, } 2 \text { participantes, avaliar efeitos de dicas usando gestos, } \\
\text { pictogramas e a fala na linguagem receptiva; GM - III; medida de satisfação não fornecida ou não } \\
\text { aplicável }\end{array}$ \\
\hline $\begin{array}{l}\text { Remington } \\
\text { Clarke, } 1983\end{array}$ & $\begin{array}{l}\text { SL; Experimental alternado, } 2 \text { participantes, comparar efeitos de da comunicação total e fala na } \\
\text { linguagem expressiva; GM - II; medida de satisfação não fornecida ou não aplicável }\end{array}$ \\
\hline $\begin{array}{l}\text { Rotholz et al., } \\
1989\end{array}$ & $\begin{array}{l}\text { SL; Experimental reversivo } \mathrm{ABAB}, 2 \text { participantes, avaliar efeitos do procedimento de espera no } \\
\text { uso de sistema pictográfico de comunicação; GM - I; medida de satisfação não fornecida ou não } \\
\text { aplicável }\end{array}$ \\
\hline $\begin{array}{l}\text { Schepis et al., } \\
1982\end{array}$ & $\begin{array}{l}\text { SP; Linha de base múltipla, } 4 \text { participantes, avaliar efeitos de procedimentos naturalísticos de } \\
\text { ensino e comunicação total na linguagem expressiva; GM - II; medida de satisfação fornecida }\end{array}$ \\
\hline $\begin{array}{l}\text { Schepis et al., } \\
1998\end{array}$ & $\begin{array}{l}\text { VOCA; Linha de base múltipla, } 4 \text { participantes, avaliar efeitos de um sistema com acionador de } \\
\text { voz + ensino naturalístico na linguagem expressiva; GM - II; medida de satisfação não fornecida } \\
\text { ou não aplicável }\end{array}$ \\
\hline $\begin{array}{l}\text { Schlosser et al., } \\
2006\end{array}$ & $\begin{array}{l}\text { VOCA; Experimental alternado, } 5 \text { participantes, avaliar efeitos de um sistema com acionador de } \\
\text { voz na linguagem expressiva; GM - II; medida de satisfação não fornecida ou não aplicável }\end{array}$ \\
\hline $\begin{array}{l}\text { Schmit et al., } \\
2000\end{array}$ & $\begin{array}{l}\text { SP; Linha de base múltipla, } 1 \text { participante, avaliar efeitos de dicas usando fala e pictogramas na } \\
\text { linguagem receptiva e comportamentos disruptivos; GM - III; medida de satisfação não fornecida } \\
\text { ou não aplicável }\end{array}$ \\
\hline $\begin{array}{l}\text { Shwartz et al. } \\
1998\end{array}$ & $\begin{array}{l}\text { SP; Estudo de caso descritivo, } 16 \text { participantes, efeitos do PECS na linguagem expressiva; } \\
\text { GM - III; medida de satisfação não fornecida ou não aplicável }\end{array}$ \\
\hline $\begin{array}{l}\text { Sigafoos et al., } \\
2004(A)\end{array}$ & $\begin{array}{l}\text { VOCA; Quase-experimental A-B, } 1 \text { participante, avaliar efeitos de um sistema com acionador de } \\
\text { voz na linguagem expressiva; GM - II; medida de satisfação não fornecida ou não aplicável }\end{array}$ \\
\hline $\begin{array}{l}\text { Sigafoos et al., } \\
2004(B)\end{array}$ & $\begin{array}{l}\text { VOCA; Linha de base múltipla, } 2 \text { participantes, avaliar efeitos de um sistema com acionador de } \\
\text { voz na linguagem expressiva; GM - III; medida de satisfação não fornecida ou não aplicável }\end{array}$ \\
\hline $\begin{array}{l}\text { Sigafoos et al., } \\
2003\end{array}$ & $\begin{array}{l}\text { VOCA; Linha de base múltipla, } 3 \text { participantes, avaliar efeitos de um sistema com acionador de } \\
\text { voz na linguagem expressiva; GM - III; medida de satisfação não fornecida ou não aplicável }\end{array}$ \\
\hline Sigafoos, 1998 & $\begin{array}{l}\text { SP; Intrassujeito reversivo, } 1 \text { participante, efeitos da aplicação de procedimentos da AEC no uso } \\
\text { de pictograma; GM - III; medida de satisfação não fornecida ou não aplicável }\end{array}$ \\
\hline Stiebel, 1999 & $\begin{array}{l}\text { SP; Linha de base múltipla, } 3 \text { participantes, efeitos do Natural Language Paradigm em treinamento } \\
\text { de pais no uso de pictogramas em crianças com autismo; GM - I; medida de satisfação fornecida }\end{array}$ \\
\hline
\end{tabular}

Quadro 1 - Características dos 56 estudos analisados. (continuação) 


\begin{tabular}{|c|c|}
\hline Autores & $\begin{array}{l}\text { Tipo de CAA, delineamento, número de participantes, variáveis, generalização/ manutenção } \\
\text { de respostas; medidas de satisfação }\end{array}$ \\
\hline oner et al., & $\begin{array}{l}\text { SP; Experimental reversivo ABAB, } 5 \text { participantes, avaliar efeitos do PECS na linguagem } \\
\text { expressiva; GM - I; medida de satisfação não fornecida ou não aplicável }\end{array}$ \\
\hline indberg, et al., & L; Intrassujeito comparativo com replicação, 2 participantes, avaliação de procedimentos AEC na \\
\hline incani, 2004 & $\begin{array}{l}\text { s do PECS e língua de sinais na } \\
\text { a }\end{array}$ \\
\hline $\begin{array}{ll}\text { aughn } & \text { e } \\
\text { orner, } 1995\end{array}$ & $\begin{array}{l}\text { uso da fala e uso de pictogramas na } \\
\text { de satisfação não fornecida ou não }\end{array}$ \\
\hline $\begin{array}{l}\text { nTetzchner et } \\
2004\end{array}$ & $\begin{array}{l}\text { nunicação total (Communication } \\
\text { III; medida de satisfação não }\end{array}$ \\
\hline $\begin{array}{l}\text { alker et al., } \\
82\end{array}$ & ação total na linguagem \\
\hline $\begin{array}{l}\text { Watters et al., } \\
1981\end{array}$ & 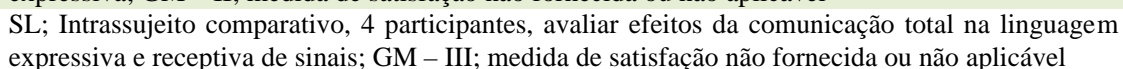 \\
\hline \multicolumn{2}{|c|}{$\begin{array}{l}\text { Legenda: Sistemas manuais e/ou língua de sinais (SL); Sistemas pictográficos (SP); sistemas assistidos com acionadores } \\
\text { de voz (VOCA); Sistemas híbridos (SH). Generalização; manutenção de respostas: Efeitos da intervenção observados } \\
\text { em pelo menos um ambiente natural (GM-I.); Generalização para outro ambiente, comportamento ou pessoa; GM-II. } \\
\text { Não avaliado ou outro (GM- III) }\end{array}$} \\
\hline
\end{tabular}

Quadro 1 - Características dos 56 estudos analisados (continuação).

Desses 56 estudos, 18 versaram sobreo uso desistemas manuais elíngua de sinais em populações com autismo; 26 empregaram sistemas pictográficos de comunicação; 9 utilizaram sistemas assistidos com acionadores de voz e 3 utilizaram sistemas híbridos, contendo mais de uma modal idade deCAA. O tipo de sistema deCAA utilizado está representado na Figura 1.

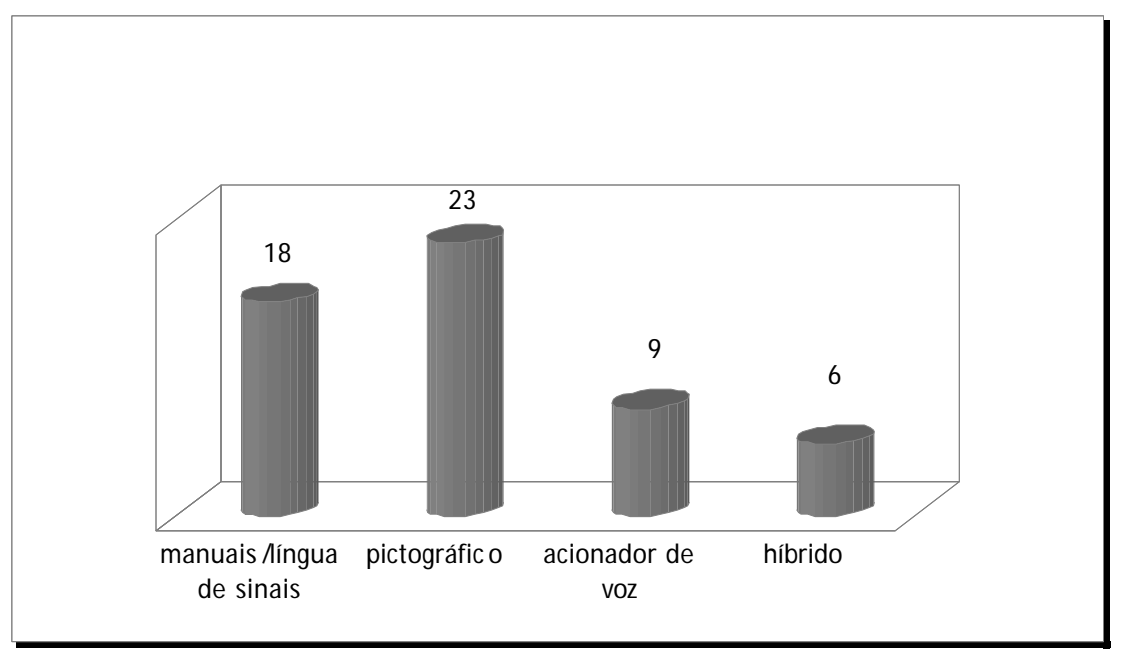

Figura 1 - Tipo de CAA. 
Ao abordar os tipos de delineamento de pesquisa, quatro trabalhos investigativos foram classificados como estudos de caso descritivos, dois tiveram como objetivo avaliar os efeitos da intervenção fazendo uso de prée de pós-testes e seis trabalharam com grupos comparativos. Os demais 44 estudos analisados fizeram uso de algum tipo de delineamento experimental ou pré-experimental do tipo sujeito-único, especialmente o delineamento do tipo linha de base múltipla, presenteem 20 pesquisas. Dezesseis trabal hos empregaram del ineamento reversivo ou tratamento al ternad o e três utilizaram delineamento do tipo A-B. Cinco estudos compararam os efeitos de diferentes procedimentos utilizando delineamento intrassujeito. O tipo de delineamento utilizado nos 56 estudos analisados está representado na Figura 2.

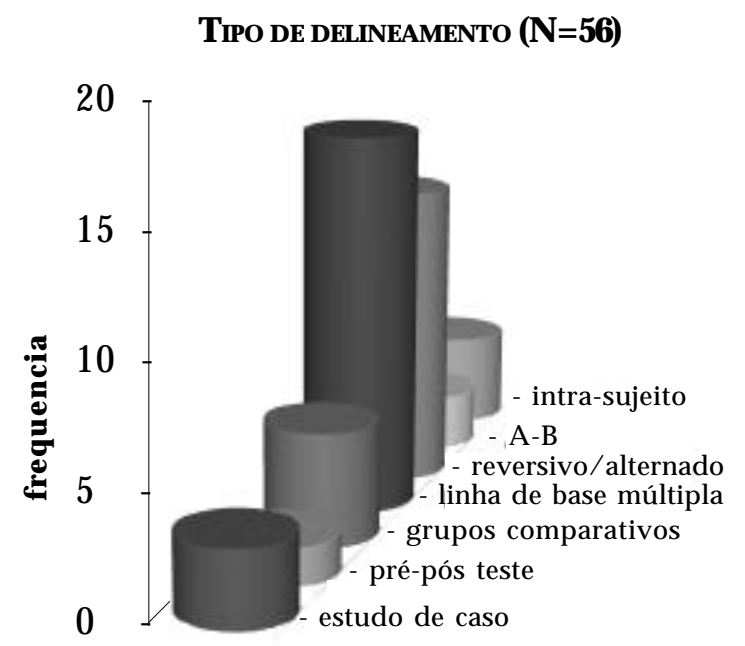

Figura 2 - Tipo de Delineamento utilizado.

Estudos que utilizam grupos de indivíduos randomicamente selecionados são raramenteencontrados em pesquisas envolvendo populações com autismo (SIMPSON, 2005). Esta mesma tendência foi observada nos trabalhos revisados, onde apenas cinco investigações utilizaram grupos compostos de 34 a 60 participantes. Em três destes trabalhos revisados os grupos foram randomicamenteselecionados (LAYTON , 1988; YODER; LAYTON , 1988; YODER; STONE, 2006). As pesquisas restantes que foram analisadas envolveram, essencialmente, um único sujeito $(\mathrm{N}=1)$ ou grupos pequenos de participantes $(\mathrm{N}<16)$, selecionados de forma incidental, sendo que os pesquisadores fizeram comparações intrassujeitos ao invés de comparações entre grupos de participantes. O número de participantes por estudo está representado na Figura 3. 


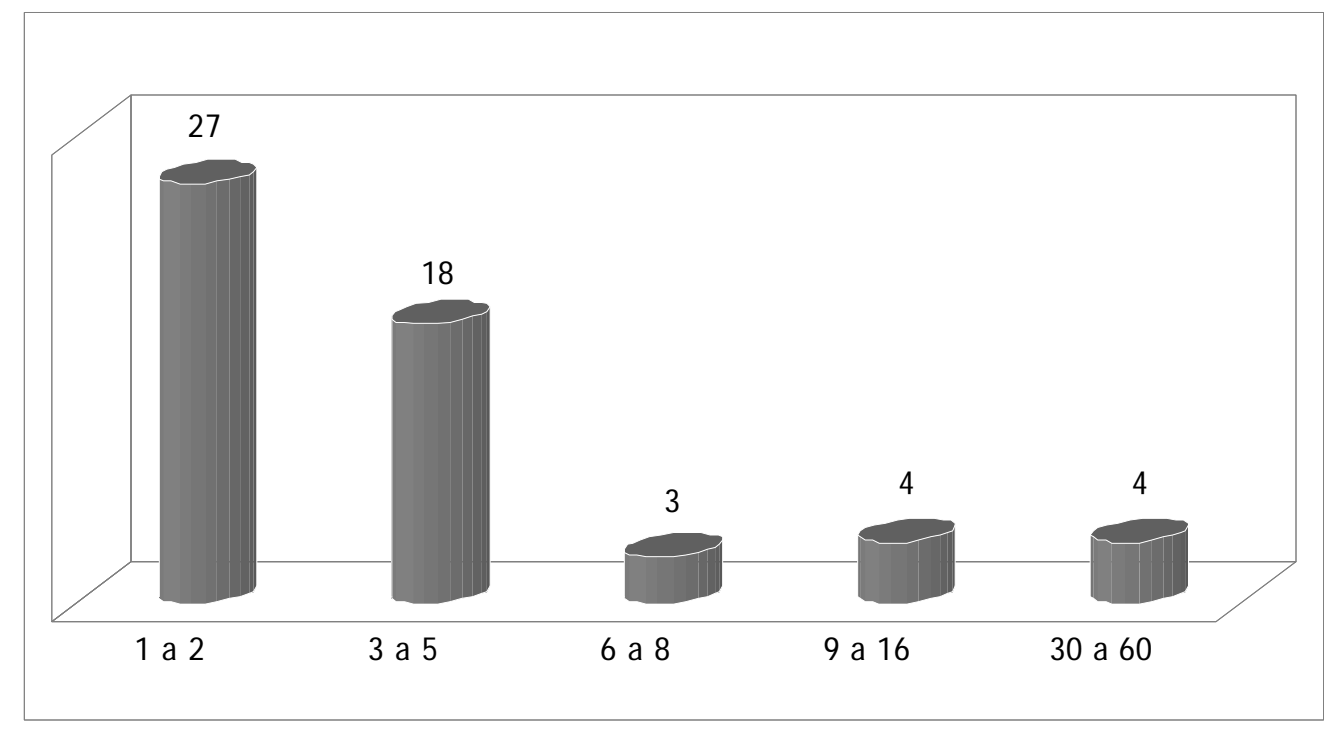

Figura 3 - Número de participantes por estudo.

A validade externa de pesquisas que empregam delineamentos de caso-único é viabilizada através da replicação de experimentos (KEN NEDY, 2005). A penas um trabalho de pesquisa analisado na presente revisão (REMINGTON; CLARKE, 1983), no entanto, implementou procedimentos experimentais idênticos aos utilizados em outra pesquisa (BA RRERA; LOBATOBARERA; SULZER-AZAROFF, 1980). Pelo menos treze pesquisas analisadas tiveram como objetivo avaliar os efeitos de um mesmo programa de intervenção (PICTURE EXCHANGE COMMUNICATIO SYSTEM - PECS), no desempenho comunicativo de indivíduos com autismo.

Em termos de validade social, metade das pesquisas revisadas $(n=28)$ descreve medidas claras de generalização de comportamentos para ambientes naturais ou sustentabilidade de respostas após a intervenção. Onze dessas investigações registraram mudanças em, pelo menos, um contexto natural. N ove estudos tiveram como objetivo avaliar o grau de satisfação dos participantes no que se refere aos efeitos produzidos pela intervenção. A frequência de estudos queevidenciam general ização ou manutenção de respostas em ambientes naturais e que, ao mesmo tempo avaliam o grau de satisfação dos participantes com os efeitos produzidos pela intervenção encontra-se disposta na Figura 4. 


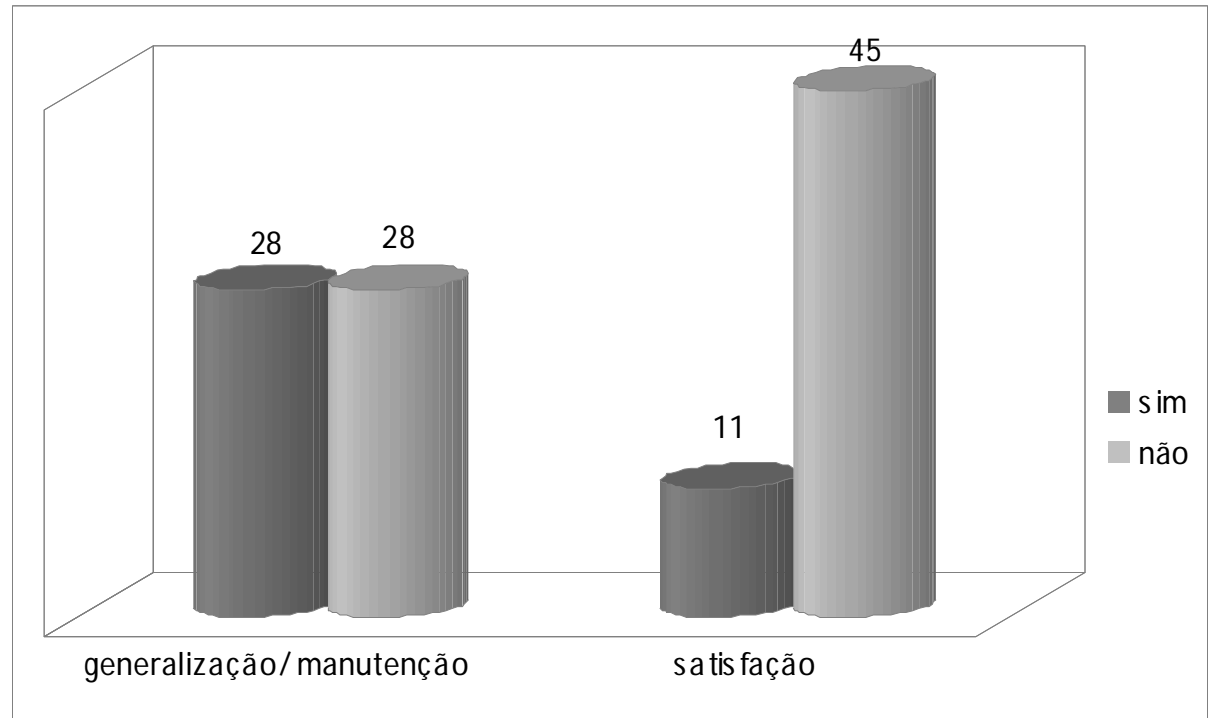

Figura 4 - Validade social: generalização/ manutenção de respostas e grau de satisfação.

\section{Conclusão}

A análise apurada das características metodológicas identificadas nos 56 artigos revisados sustenta a predominância de del ineamentos experimentais e quase-experimentais do tipo intrassujeitos, o quecorrobora a escassez de pesquisas de grupo em população de autistas. As causas apontadas, na literatura, para a ocorrência deste viés esbarram na ética da pesquisa com Seres Humanos etambém nas rotinas operacionais dos experimentos científicos. A exemplo disto, a alternativa metodológica de utilização de grupo controle e de grupo experimental para se pesquisar sobreautismo éassunto de natureza ética, quando constatados progressos para os participantes do grupo experimental, em contraste com a falta de ganhos para os participantes do grupo controle.

Neste cenário investigativo, as barreiras operacionais observadas nas pesquisas científicas sobre Autismo ocorrem quando setenta reunir, por exemplo, um número expressivo de participantes para comporem amostras homogêneas e representativas do universo pesquisado, conforme preconiza o model o estatístico eisso nem sempreéviável. Paradoxal mente, na perspectiva dos delineamentos de caso único, a homogeneização da amostra não é tão necessária, considerando-se queos erros demedida assumem outro significado nas pesquisas destetipo, quando se pretende monitorar, por exemplo, a variabilidade de respostas da pessoa com autismo. 
Os resultados das análises dos artigos revelaram também que a validade social das pesquisas sobre Autismo é evidenciada pela sustentabilidade de respostas após a fase de intervenção, considerando-se também a possibilidade de generalização e manutenção desses efeitos, em ambiente natural. Como complemento da validade social dessas pesquisas épossível determinar o grau de satisfação observado nos participantes que foram impactados pel os procedimentos de intervenção a que estiveram submetidos, ou seja a atitude favorável dos consumidores da pesquisa frente aos resultados obtidos.

Finalmente, ao serem traçadas diretrizes que favoreçam o desenvolvimento de projetos válidos esocialmente relevantes para o trabal ho com autistas, caberá aos especialistas se apropriarem das melhores práticas, ou seja, dos modos de intervenção, em Educação Especial, mais eficazes e eficientes que assegurem um desempenho ótimo do aluno, conforme sustentado por Spaulding (2009). A crescente-se a estas diretrizes os projetos de avaliação permanente nos moldes dos que se conhece na literatura como accountabil lity. N este sentido, já são conhecidos os parâmetros que tornam possivel identificar o que funciona e o que deixa de funcionar, no atendimento da pessoa com autismo, podendo-se assim decidir por modalidades de intervenção cujos resultados finais sejam notoriamente confiáveis inclusive para subsidiar as ações das políticas públicas de educação (CAMPOS, 2009).

\section{REFERÊNCIAS}

BARRERA, R.; SULZER-AZAROFF, B. An alternating treatment comparison of oral and total communication training programs with echolal ic autistic children.J ournal of A pplied Behavior A nalysis, v.16, p. 379-394, 1983.

BARRERA, R.; LOBATO-BARERA, D.; SULZER-AZAROFF, B. A simultaneous treatment comparison of three expressive language training programs with a mute autistic child. Journal of A utism and Developmental Disorders, v.10, p.21-37, 1980.

BARTMAN, S.; FREEMAN, N. Teaching language to a two-year-old with autism. Journal on Developmental Disabilities, v.10, n. 1, p.47-53, 2003.

BERNARD-OPIZ, V.; ROSS, K.; TUTTAS, M.L. Computer assisted instruction for autistic children. A nnals of the A cademy of M edicine, v. 19, p. 611-616, 1990.

BEST, J.W.; KAHN, J.V. Resarch in Education. New York: Pearson, 2006.

BONDY, A.; FROST, L. The Picture Exchange Communication System. Focus on A utistic Behavior, V.9, p.1-19, 1994.

BOSSELER, A.; MASSARO, D. Development and evaluation of a computer-animated tutor for vocabulary and language learning in children with autism. Journal of Autism and Developmental Disorders, v. 33, n. 6, p. 653-72, 2003.

BRYAN, L. C.; GAST, D. L. Teaching on-task and on-schedule behaviors to high-functioning children with autism via picture activity schedules. Journal of A utism and Developmental Disorders, v. 30, n.6, p. 553-567, 2000 
BUFFINGTON, D. M. et al. Procedures for teaching appropriate gestural communication skills to children with autism. Journal of A utism and D evelopmental Disorders, v. 28, p.535-546, 1998.

CAFIERO, J. The effect of an augmentative communication intervention on the communication, behavior and academic progress of an adolescent with autism. Focus on Autism and 0 ther Developmental Disabilities, v.16, n.3, p.179-189, 2001.

CAMPOS, M.M. Para que serve a pesquisa em educação? Cadernos de Pesquisa. v.39, n.136, São Paulo. Jan/ A pr, 2009.

CARR, E.; DORES, P.A. Patterns of language acquisition following simultaneous communication with autistic children. Analysis and Intervention in Developmental Disabilities, v.1, p.347-361, 1981.

CARR, E.; KOLOGINSKY, E. A cquisition of sign language by autistic children. II: Spontaneity and generalized effects. Journal of A pplied Behavior Analysis, v.16, n.3, p. 297-314, 1983.

CARR, E.; KOLOGINSKY, E.; LEFF-SIMON, S. Acquisition of sign languageby autistic children. III: Generalized descriptive phrases. Journal of A utism and D evelopmental Disorders, v.17, n.2, p. 217-229, 1987.

CARR, E.; PRIDAL, C.; DORES, P. Speech versus sign comprehension in autistic children: analysis and prediction. Journal of Experimental Child Psychology, v.37, p. 587-597, 1984.

CHARLOP-CHRISTY, M. H., et al. Using the picture exchange communication system (PECS) with children with autism: Assessment of PECS acquisition, speech, social-communicative behavior, and problem behavior. Journal of A pplied Behavior Analysis, v.35, p. 213-231, 2002.

COZBY, P. C. Métodos de pesquisa em ciências do comportamento. São Paulo: Atlas, 2003.

CUMMINGS, A. R.,; WILLIAMS, W. L. Visual identity matching and vocal imitation training with children with autism: A surprising finding. J ournal on D evelopmental D isabilities, v.7, p.123-141, 2000.

DYCHES, T. Effects of switch training on the communication of children with autism and severe disabilities. Focus on A utism and O ther D evelopmental Disabilities, v.13, p. 151-162, 1998.

FERRA RESE, R.; NORTON , P. ; WHITM ONT, S. Can signing improvethequality of autistic speech? The Exceptional Child, v.29, p. 117-125, 1982.

FREA, W.; ARN OLD, C.; VITTIMBERGA, G. A demonstration of the effects of augmentative communication on the extreme aggressive behavior of a child with autism within an integrated preschool setting. Journal of Positive Behavior Intervention, v.3, p. 194-198, 2001.

GANZ J.B.; SIMPSON, R.L. Effects on communicative requesting and speech development of the Picture Exchange Communication System in children with characteristics of autism. J ournal of A utism and D evelopmental Disorders, v. 34, n.4, p. 395-409, 2004.

GANZ, J. B.; SIMPSON, R. L.; CORBIN-NEWSOME, J. The impact of the Picture Exchange Communication system on requesting and speech development in preschoolers with autism spectrum disorders and similar characteristics. Research in A utism Spectrum Disorder, no prelo.

GIL, A.C. Como elaborar projeto de pesquisa. São Paulo: Atlas, 2007

HAMILTON, B.; SNELL, M. Using the Milieu Approach to increase spontaneous communication book use across environments by an adolescent with autism. Augmentative and Alternative Communication, v.9, p. $259-272,1993$.

KEEN, D.; SIGAFOOS, J.; WOODYATT, G. Replacing prelinguistic behaviours with functional communication. Journal of A utism and Developmental Disorders, v.31, n.4, p. 385-398, 2001.

KENNEDY, C.H. Single-case designs for educational research. Boston: Pearson Education, Inc, 2005. 
KOURI, T. Effects of simultaneous communication in a child-directed treatment approach with preschoolers with severe disabilities. A ugmentative and Alternative Communication, v.4, n.4, p. 222232, 1988.

KOZLESKI, E. Visual symbol acquisition by students with autism. Exceptionality, v.2, p. 173-194, 1991.

KRAVITS, T. et al. Brief report: Increasing communication skills for an elementary-aged student with autism using the Picture Exchange Communication System. Journal of A utism and D evel opmental Disorders, v.32, p. 225-230, 2002.

LAYTON, T. Language training with autistic children using four different modes of presentation. Journal of Communication Disorders, v.21, p. 333-350, 1988.

LIGHT J. et al. Augmentative and alternative communication to support receptive and expressive communication for people with autism. Journal of Communication Disorder, v 31, p. 153-180, 1998.

MACDUFF, G. S.; KRANTZ, P.J.; MCCLANNAHAN, L. E. Teaching children with autism to use photographic activity schedules: $M$ aintenance and generalization of complex responsechains. Journal of A pplied Behavior A nalysis, v.26, p. 89-95, 1993.

MAGIATI I.; HOWLIN P. A pilot evaluation study of the Picture Exchange Communication System (PECS) for children with autistic spectrum disorders. Autism, v.7, n.3, p. 297-320, 2003.

MARCKEL, J.; NEEF, N. A.; FERRERI, S. J. A preliminary analysis of teaching improvisation skills with thePicture Exchange Communication System to children with autism. Journal of A pplied Behavior A nalysis, v. 39, p. 109-115, 2006.

MORAIS, E. M. U m estudo sobre a validade e fidedignidade de métodos de avaliação de interfaces. 2007. 114f. Dissertação (Mestrado em Ciência da Computação) - Programa de Pós-Graduação em Ciência da Computação, Universidade Estadual de Maringá, Maringá, 2007.

NATIONAL RESEARCH COUNCIL. Educating children with autism. Committee on Educational Interventions for Children with Autism. Catherine Lord and James McGee (Eds). Division of Behavioral and Social Sciences and Education. Washington, DC : N ational A cademy Press, 2001.

NUNES, L.R. et al. Pesquisa em educação especial na pós-graduação. In: Questões A tuais em Educação Especial - Volume III, Rio de Janeiro: Sete Letras, 1998.

NUNES, D. AAC Interventions for autism: A research summary. International Journal of Special Education. v.23, p.17 - 26, 2008.

NUNES, D.; HANLINE, M. Enhancing the AAC use of a child with autism through a parentimplemented naturalistic intervention. International Journal of Disability, Development and Education, v.54, p.177-197, 2007.

NUNES SOBRIN HO, F., NAUJORKS, M. I. Pesquisa em educação especial: o desafio da qualificação. Bauru, São Paulo: Ed. EDUSC, 2001.

O'NEILL, R. E.; SWEETLAND-BAKER, M. Brief report: An assessment of stimulus generalization and contingency effects in functional communication training with two students with autism. Journal of Autism \& Developmental Disorders, v. 31, n. 2, p. 235-240, 2001.

PARSONS, C.; LA SORTE, D. The effect of computers with synthesized speech and no speech on the spontaneous communication of children with autism. A ustralian Journal of Communication Disorders, v. 21, n.1, p. 12-31, 1993.

PETERSON, S. et al.. Effects of alternating communication input for students with autism and no speech: two case studies. A ugmentative and A Iternative Communication, v.11, p.93-100, 1995. 
REM IN GTON, B.; CLARKE, S. A cquisition of expressive signing by autistic children: an evaluation of the relative effects of simultaneous communication and sign alone training. Journal of A pplied Behavior A nalysis, v. 16, n.3, p. 315-328, 1983.

ROTHOLZ, D. A.; BERKOWITZ, S.F.; BURBERRY, J. Functionality of two modes of communication in the community by students with developmental disabilities: a comparison of signing and communication books. Journal of the A ssociation of Persons with Severe H andicaps, v.14, n.3, p. 227-233, 1989.

SCHEPIS, M. et al. A program for increasing manual signing by autistic and profoundly retarded youth within the daily environment. Journal of A pplied Behavior A nalysis, v.15, n.3, p. 363-379, 1982.

SCHEPIS, M. et al. Increasing communicative interactions of young children with autism using a voice output communication aid and naturalistic teaching. Journal of A pplied Behavior A nalysis, v.31 p. 561-578, 1998.

SCHLOSSER, R. et al. Effects of synthetic speech output on requesting and natural speech production in children with autism. Research in A utism Spectrum Disorders, v.1, p.139-163, 2007.

SCHMITT, J. et al. The effects of using a photographic cueing package during routine school transitions with a child with autism. M ental Retardation, v.38, n.2, p. 131-137, 2000.

SCHWARTZ, I.; GARGINKLE, A.; BAUER, J. The picture exchange communication system: communicative outcomes for young children with disabilities. Topics in Early Childhood Special Education, v.,8, n.3, p.144-159, 1998.

SIGAFOOS, J. et al.Teaching VOCA use as a communication repair strategy. Journal of Autism and D evelopmental D isorders, v.34, p. 411-422, 2004 (a).

SIGAFOOSJ, et al. Transferring AAC intervention to the home. D isabil Rehabil. v.26, p. 1330-4, 2004 (b).

SIGAFOOS, J. Assessing conditional use of graphic mode requesting in a young boy with autism Journal of D evelopmental and Physical Disabilities, v.10, p. 133 - 151, 1998.

SIGAFOOS et al. Effects of voice-output technology on requesting and vocalizations in threechildren with developmental disabilities. A ugmentative and A Iternative Communication, v. 19, p. 37-47, 2003.

SIMPSON, R. Evidence-based practices and students with autism spectrum disorders. Focus on A utism and Other Developmental Disabilities, v. 20, n.3, p. 140-149, 2005.

SPAULDING, L. Best practices and interventions in Special Education: How do we know what works? Teaching Exceptional Children Plus, v. 5, n.3, p. 1-13, 2009.

STIEBEL, D. Promoting augmentative communication during daily routines: a parent problemsolving intervention. Journal of Positive Behavior Interventions v.1, n.3, p. 159-169, 1999.

STONER. J.B. The effectiveness of the Picture Exchange Communication System with nonspeaking adults. Remedial and Special Education, v. 27, n. 3, p. 154-165, 2006.

SUNDBERG, M. L.; ENDICOTT, K; EIGENHEER, P. Using intraverbal prompts to establish tacts for children with autism. The A nalysis of V erbal Behavior, v.17, p. 89-104, 2000.

TINCANI, M. Comparing the Picture Exchange Communication System and sign language training for children with autism. Focus on A utism and O ther D evelopmental Studies, v.19, p. 152-163, 2004.

VAUGHN, B.; HORNER, R. Effects of concrete versus verbal choice systems on problem behavior. A ugmentative and A Iternative Communication, v. 11, p. 89-92. 1995.

VONTETZCHNER, S. et al. Acquisition of graphic communication by a young girls without comprehension of spoken language. Disability and Rehabilitation, v. 26, n. 2, p. 1335-1346, 2004. 
NUNES, D.R.P.; NUNES SOBRINHO, F.P.

WALKER, G.R. et al. Sign language as a prompt to teach a verbal "yes" and "no" discrimination to an autistic boy. Child Behavior Therapy, v. 3, n. 4, p. 77-86, 1982.

WATTERS, R.; WHEELER, L.; WATTERS, W. The relative efficiency of two orders for training training autistic children in the expressiveand receptive use of manual signs. Journal of Communication Disorders, v. 14, p. 273-285, 1981.

WHERRY, J.; EDWARDS, R. A comparison of verbal, sign, and simultaneous system for the acquisition of receptive language by an autistic boy. Journal of Communication D isorders, v. 16, p. 201216, 1983.

WOLF, M. M. Social validity: The case for subjective measurementor How applied behavior analysis is finding its heart. Journal of A pplied Behavior A nalysis, v. 11, p. 203-214, 1978.

YODER, P.; LA YTON, T. Speech following sign language training in autistic children with minimal verbal language. Journal of Autism and D evelopmental Disorders, v. 18, p. 217 - 229, 1988.

YODER, P.; STONE, W. Randomized comparison of two communication interventions for preschoolers with autism spectrum disorders. Journal of Consulting and Clinical Psychology. v. 74, n.3, p. 426-435, 2006

Recebido em: 06/ 10/ 2009

Reformulado em: 06/ 03/ 2010

A provado em: 08/ 05/ 2010 